\section{Fortgeschrittene COPD: Inhalative Triple-Therapie verhindert Exazerbationen}

Papi A et al. Extrafine inhaled triple therapy versus dual bronchodilator therapy in chronic obstructive pulmonary disease (TRIBUTE): a double-blind, parallel group, randomised controlled trial. Lancet 2018; 391: 1076 - 1084

Die zusätzliche Gabe eines inhalativen Kortikosteroids zu einer dualen bronchodilatatorischen Therapie ist bei der COPD umstritten. Eine randomisierte Studie zeigte jetzt, dass eine solche Triple-Therapie im Vergleich zu einer dualen bronchienerweiternden Behandlung Exazerbationen effektiver verhindert, ohne dass häufiger Pneumonien auftreten.

In die multizentrische, prospektive, randomisierte, doppelt-verblindete TRIBUTE-Studie wurden zwischen 2015 und 2017 insgesamt 1532 Patientinnen und Patienten $>40$ Jahre mit fortgeschrittener COPD aufgenommen. Lungenfunktionstechnische Einschlusskriterien waren ein $\mathrm{FEV}_{1} / \mathrm{FVC}$-Verhältnis <0,7 nach Bronchospasmolyse mit Salbutamol sowie eine $\mathrm{FEV}_{1}<50 \%$ der Norm. Innerhalb der letzten 12 Monate musste es darüber hinaus zu mindestens einer moderaten bis schweren Exazerbation der COPD gekommen sein und die Patienten trotz einer bronchodilatatorischen Therapie mit oder ohne Kortison (nicht aber mit einer Triple-Therapie) noch symptomatisch sein. Anhand des FEV -Wertes wurde $_{1}$ eine stratifizierte 1:1-Randomisierung in folgende Therapiegruppen vorgenommen:

- 1-mal tgl. Pulverinhalation eines Kombinationspräparates bestehend aus $85 \mu \mathrm{g}$ Indacaterol (langwirksames Beta-2-Mimetikum) und $43 \mu \mathrm{g}$ Glycopyrronium (Parasympatholytikum)

- 2-mal tgl. Inhalation eines Kombinationspräparates bestehend aus $87 \mu \mathrm{g}$ Beclometason (Kortikosteroid), $5 \mu \mathrm{g}$ Formoterol (Beta-2-Sympathomimetikum) und $9 \mu \mathrm{g}$ Glycopyrronium; Formulierung mit besonders kleiner Partikelgröße
Die Behandlung war jeweils für einen Zeitraum von 52 Wochen vorgesehen, als zusätzliche Bedarfsmedikation waren Salbutamol bzw. Terbutalin zugelassen. Primärer Endpunkt war die Anzahl moderater bis schwerer Exazerbationen während des Studienzeitraums.

\section{Exazerbationsrate um 15\% verringert}

Beide Behandlungsgruppen zeichneten sich durch eine hohe Therapie-Compliance aus; es wurden von den Patienten jeweils $98 \%$ der vorgesehenen Applikationen durchgeführt. In der Triple-Therapie-Gruppe lag die Rate der moderaten bis schweren Exazerbationen bei 0,5\% pro Patient und Jahr. Demgegenüber wurden unter der dualen Behandlung 0,59\% Exazerbationen pro Patient und Jahr verzeichnet. Es errechnete sich eine Reduktion der Exazerbationsrate um 15\% unter der Inhalation von BeclometasonFormoterol-Glycopyrronium gegenüber der Anwendung von Indacaterol-Glycopyrronium. Unter der Dreifachtherapie erfuhren die Patienten außerdem eine frühere Symptombesserung und gaben eine höhere gesundheitsbezogene Lebensqualität an als Patienten, die die Zweifachtherapie anwendeten.

In beiden Behandlungsgruppen traten bei $4 \%$ der Patienten Pneumonien auf (>80\% radiologisch gesichert). Auch die Rate kardialer Nebenwirkungen war unter beiden Therapien vergleichbar (6 bzw. 7\%).

FAZIT

Symptomatische Patienten mit fortgeschrittener COPD profitierten von der 1-mal täglichen inhalativen TripleTherapie mit einem neuen Kombinationspräparat aus Beclometason, Formoterol und Glycopyrronium. Dabei wurde im Gegensatz zu vorausgegangenen Studien durch die inhalative Kortisongabe kein zusätzliches Risiko für Pneumonien verzeichnet.

Dr. Katharina Franke, Darmstadt 